Review began 01/10/2022 Review ended 02/01/2022 Published 02/05/2022

๑) Copyright 2022

Blotner et al. This is an open access article distributed under the terms of the Creative Commons Attribution License CC-BY 4.0. which permits unrestricted use, distribution, and reproduction in any medium, provided the original author and source are credited.

\section{Workup for Suspected Brugada Syndrome: Two Case Reports for the General Practitioner}

\author{
Michael Blotner ${ }^{1}$, Omkar Betageri ${ }^{1}$, William Miles ${ }^{2}$, Kun Xiang ${ }^{2}$ \\ 1. Internal Medicine, University of Florida College of Medicine, Gainesville, USA 2. Cardiology, University of Florida \\ College of Medicine, Gainesville, USA
}

Corresponding author: Michael Blotner, michael.blotner@medicine.ufl.edu

\begin{abstract}
While a large proportion of ST-segment elevation on EKG is related to myocardial ischemia, the differential diagnosis must include pericarditis, channelopathies, and various genetic conditions. Identifying and working up such abnormalities present a challenge to primary care providers (PCPs). We present two clinical cases of young male patients with ST-segment elevation in anteroseptal leads suspicious for Brugada syndrome and show how to risk stratify and manage them.
\end{abstract}

Our first case presents a 23-year-old male with no past medical history with acute onset substernal chest pain, shortness of breath, and palpitations. Initial workup revealed negative serial troponins and normal Btype natriuretic peptide (BNP). The EKG revealed ST elevation in lead V2. An evaluation for Brugada syndrome was pursued. Upon completion of a procainamide challenge, it was determined that he did not have Brugada syndrome and was shortly discharged.

Our second case presents a 33-year-old male with no pertinent cardiac medical history who presented to an outpatient cardiology clinic after discovering an incidental ST elevation in V2 on EKG. His family history was negative for early atherosclerotic cardiovascular events or sudden cardiac death. The patient's initial workup was negative. Suspicion for Brugada syndrome leads to performing a procainamide challenge, which was significant for ST changes in the anterolateral leads. He was asymptomatic during the challenge and initial presentation, and no further intervention was indicated. He was advised to avoid sodium channel blocking medications and treat any fevers and was sent for genetic testing.

These cases illustrate the importance of maintaining an appropriate suspicion for Brugada syndrome in young patients with minimal ischemic risk factors. We discuss a guideline-directed algorithmic workup for PCPs in suspicious individuals. Stratifying patients based on the presence of symptoms, history of tachyarrhythmias, and EKG findings before and after drug challenge allows physicians to guide further management of these patients.

Categories: Cardiology, Family/General Practice, Internal Medicine

Keywords: ventricular arrhythmia, brugada electrocardiogram pattern, brugada phenocopy, procainamide, stelevation, brugada syndrome, brugada

\section{Introduction}

One of the most common diagnostic tools in both the hospital and ambulatory setting for general practitioners is the use of an EKG. ST-segment elevation on EKG can increase suspicion for myocardial infarction (MI), but other causes may include pericarditis, early repolarization, left ventricular hypertrophy, left bundle branch block, ventricular aneurysm, or takotsubo cardiomyopathy [1,2]. One additional cause of ST-segment elevation is Brugada syndrome, which is associated with ventricular arrhythmia and sudden cardiac death [3-6]. EKGs in patients with Brugada syndrome have a characteristic ST-segment elevation in the anterolateral leads (V1 to V3) [4,6-8]. It poses a challenge to primary care clinicians to assess the risks of sudden cardiac death in such patients. Here, we present two clinical cases of patients with ST-segment elevation in anteroseptal leads and provide fundamental suggestions on risk stratification and management.

\section{Case Presentation}

\section{Case 1}

A 23-year-old male with no significant past medical or family history presented to the emergency department for acute onset substernal chest pain, shortness of breath, and palpitations that occurred 30 minutes after putting out a small kitchen fire amidst consumption of alcohol. When emergency medical service (EMS) arrived at the scene, the patient was diaphoretic and in severe respiratory distress. On arrival at the emergency department, his symptoms had resolved. Initial workup was significant for negative serial troponins, B-type natriuretic peptide (BNP) within normal limits, and lactic acid of $5.2 \mathrm{mmol} / \mathrm{L}$. EKG obtained is shown in Figure 1. 


\section{Cureus}

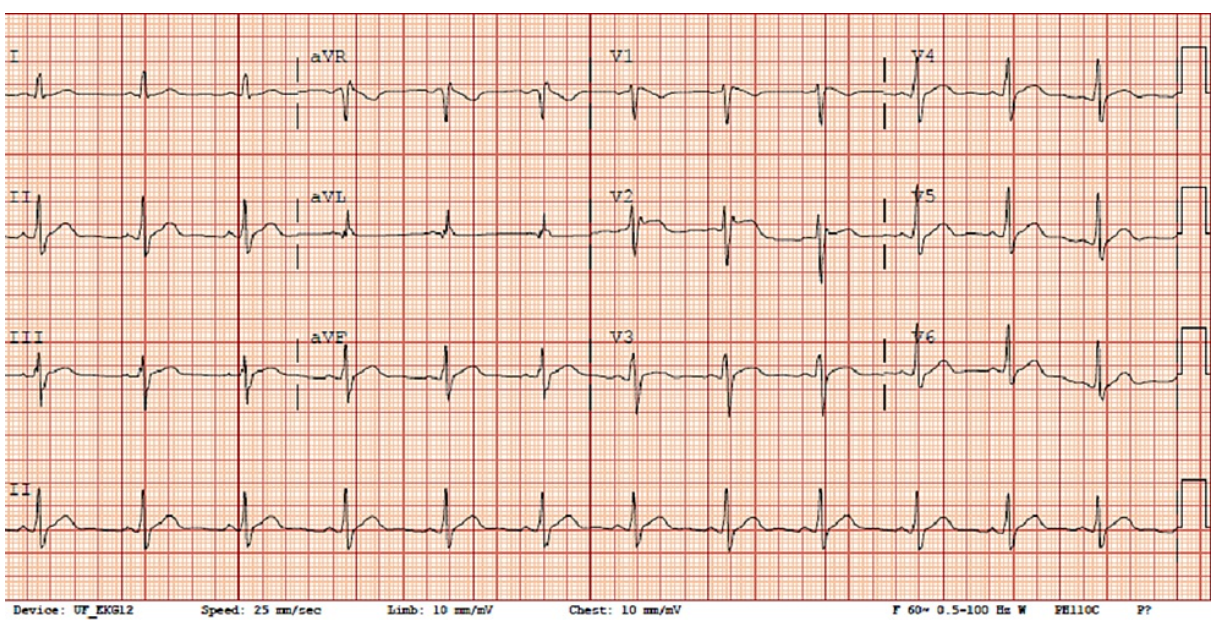

FIGURE 1: Baseline EKG in the emergency department.

The patient was then admitted to the hospital's cardiology service for further workup and management. During the admission, there was a concern for Brugada syndrome, given the morphology of the above EKG in V2. Echocardiogram performed showed no abnormalities. The patient was taken for an IV procainamide challenge. The resulting EKG after a full 1,000 mg challenge is shown in Figure 2.

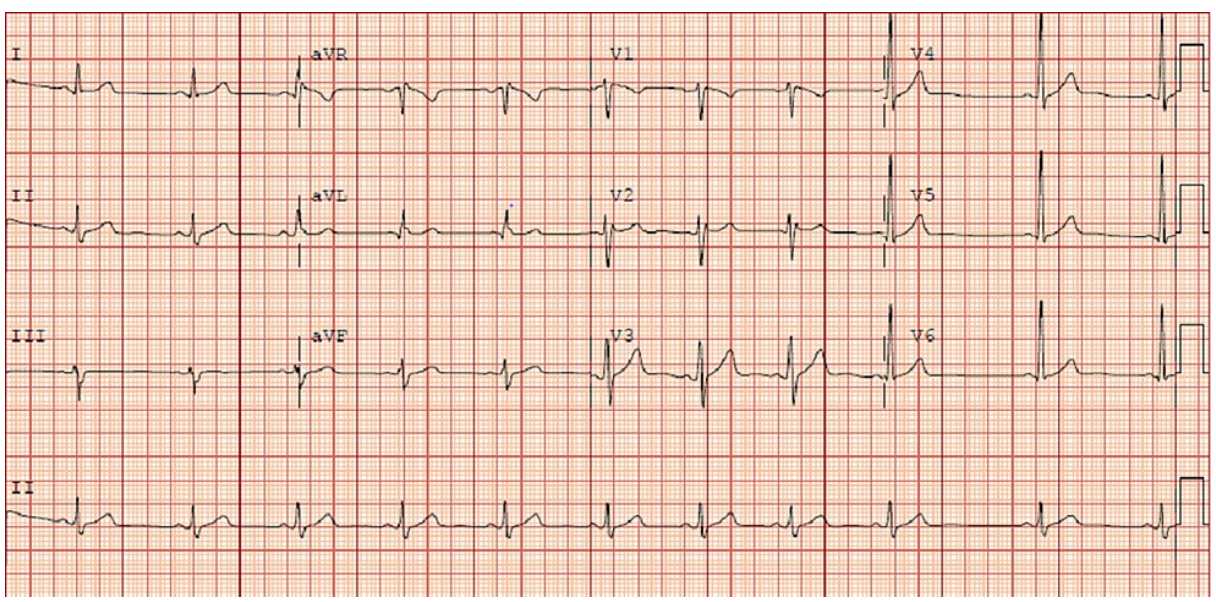

FIGURE 2: EKG obtained after procainamide challenge.

Given the patient's unchanged EKG with a pharmacologic challenge along with atypical symptoms for Brugada syndrome, the patient was discharged home without further workup or management.

\section{Case 2}

A 33-year-old male with a past medical history of attention deficit hyperactivity disorder (ADHD) was referred to our cardiology office for an abnormal EKG before refilling his methylphenidate prescription. He reports one occurrence of panic attacks in the past year, where he had palpitations and diaphoresis before an important exam. He denies any history of syncope or pre-syncope. Family history is notable for a father with a $4.8 \mathrm{~cm}$ ascending aortic aneurysm and a paternal uncle that died from an aortic dissection. There is no family history of sudden death or syncope. He previously had a 24-hour Holter monitor and echocardiogram that were unremarkable. Overall, the patient felt well and denied any chest pain, shortness of breath, palpitations, or orthopnea. Referral EKG is shown in Figure 3. 


\section{Cureus}

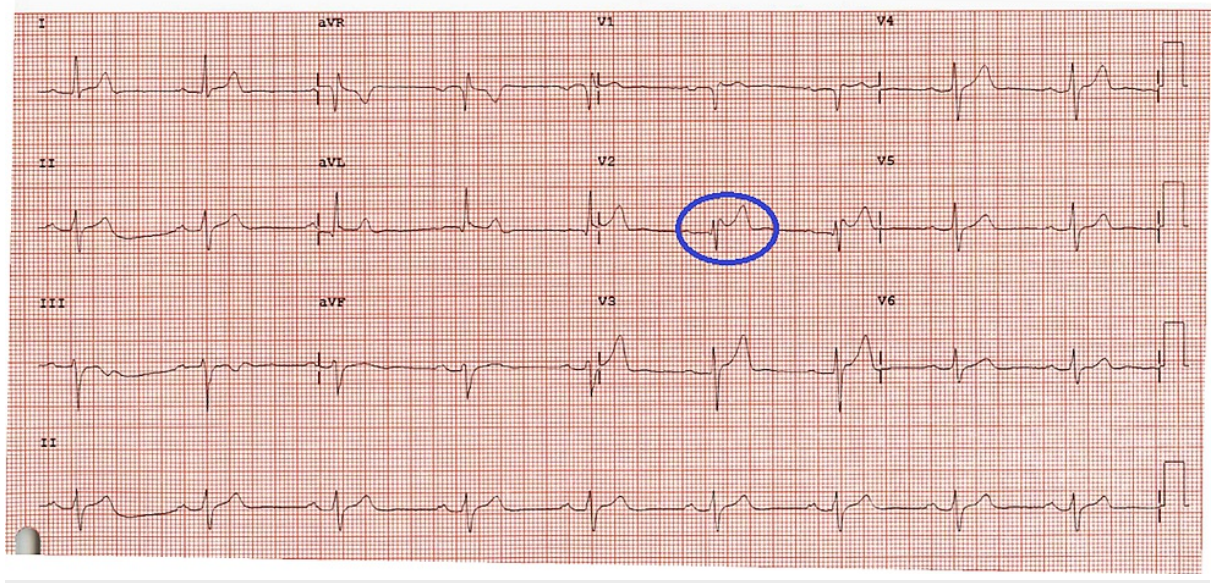

FIGURE 3: Baseline EKG showing Brugada pattern type 2 with a saddleback appearance in V2.

Given the concern for Brugada syndrome based on the findings in V1-V2, the patient underwent a procainamide challenge with the following EKGs shown in Figures 4, 5.

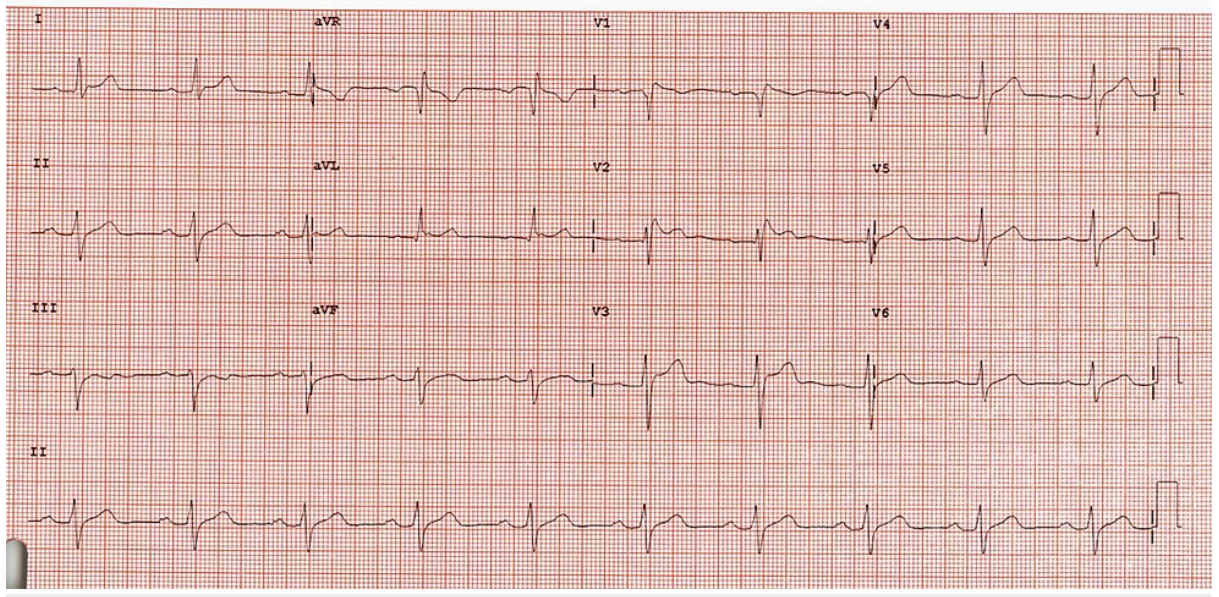

FIGURE 4: EKG at minute 14 of procainamide infusion $(700 \mathrm{mg})$ showing the conversion from Brugada pattern type 2 to type 1 . 


\section{Cureus}

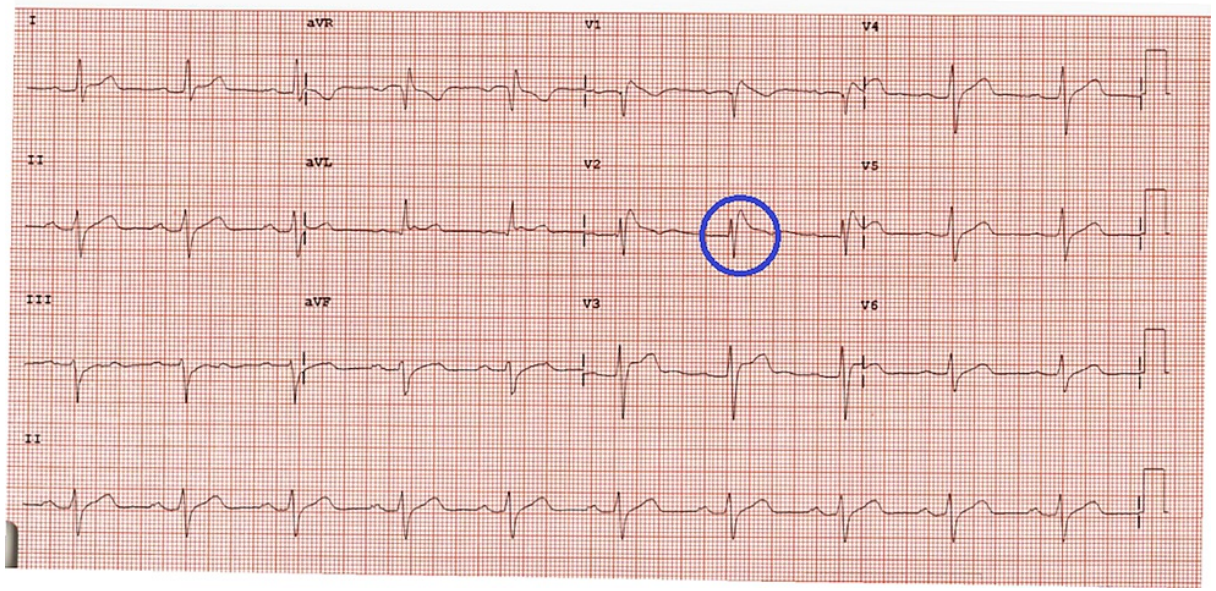

\section{FIGURE 5: EKG at minute 20 of procainamide $(1,000 \mathrm{mg})$ showing}

Brugada pattern type 1 with coved ST-segment elevation in V1-V2.

The above EKGs show the conversion of the type 2 Brugada pattern to the type 1 Brugada pattern. This confirms the diagnosis of Brugada syndrome. Due to the patient's lack of family history or personal history of syncope or tachyarrhythmia, no further workup or intervention was indicated. However, genetic tests were sent and the patient was advised that first-degree relatives should obtain screening EKGs. He was also advised to avoid drugs with sodium channel blocking activity and was made aware that fever can exacerbate arrhythmias in Brugada syndrome.

\section{Discussion}

Brugada syndrome is an autosomal dominant disorder with a variable expression that presents with abnormal EKG findings in conjunction with an increased incidence of ventricular tachyarrhythmia and sudden cardiac death [9]. The clinical disease has a male predominance, with an average age of diagnosis at 41 years old $[10,11]$. Primary care providers (PCPs) serve a vital role in the prevention of cardiac disease. In both the adult and pediatric domains, it is recommended that PCPs focus cardiac screening on risk factors and family history of any cardiac abnormalities $[6,12,13]$. The American Academy of Pediatrics specifically recommends screening for family history of sudden cardiac death or sudden cardiac arrest for primary prevention [6]. In particular, the PCP is especially critical in the context of Brugada syndrome, as multiple family members within their patient panels may be affected. Current recommendations include screening all first-degree relatives of any patient diagnosed with Brugada syndrome [9].

Our cases serve as fundamental examples of the workup algorithm of suspected Brugada syndrome, which may be encountered by the PCP. Both cases presented young men with no prior cardiac history with EKG findings consistent with Brugada pattern type 2. Prompt and accurate diagnosis of Brugada syndrome is critical, as symptomatic patients have an elevated risk of sudden cardiac death from arrhythmia [5]. While the prevalence of Brugada syndrome in patients with Brugada pattern EKG is not well established, some studies estimate a $10 \%$ event rate at 2.5 years [3].

In case 1 , the morphology of the ST segment continues to have the saddleback appearance of a type 2 pattern throughout the procainamide challenge and does not change. This is classified as a negative pharmacological challenge, and therefore no further workup was indicated for the patient. In contrast, case 2 was noted to have a conversion from the type 2 to type 1 Brugada pattern with the procainamide challenge. No ventricular arrhythmia was induced. Although case 2 confirmed a diagnosis of Brugada syndrome, an absence of a cardiac arrest, tachyarrhythmia, or syncope in his history indicates that he does not require further workup. This is based on stratification data, which demonstrate that an inducible type 1 pattern in the absence of prior symptoms or inducible arrhythmia has an elevated but overall low chance of developing a ventricular arrhythmia (Figure 6 ) $[5,7]$. However, given that he does have a positive diagnosis, he was evaluated for genetic components of Brugada syndrome, and his first-degree relatives were advised to seek evaluation for Brugada syndrome. He was also advised to avoid medications that inhibit cardiac sodium channels (class I antiarrhythmics, tricyclic antidepressants (TCAs), and lithium) in the future, as this would risk inducing an arrhythmia. Another consideration in Brugada syndrome patients is prompt treatment of any fevers with antipyretics for arrhythmia prevention [14]. 


\section{Cureus}

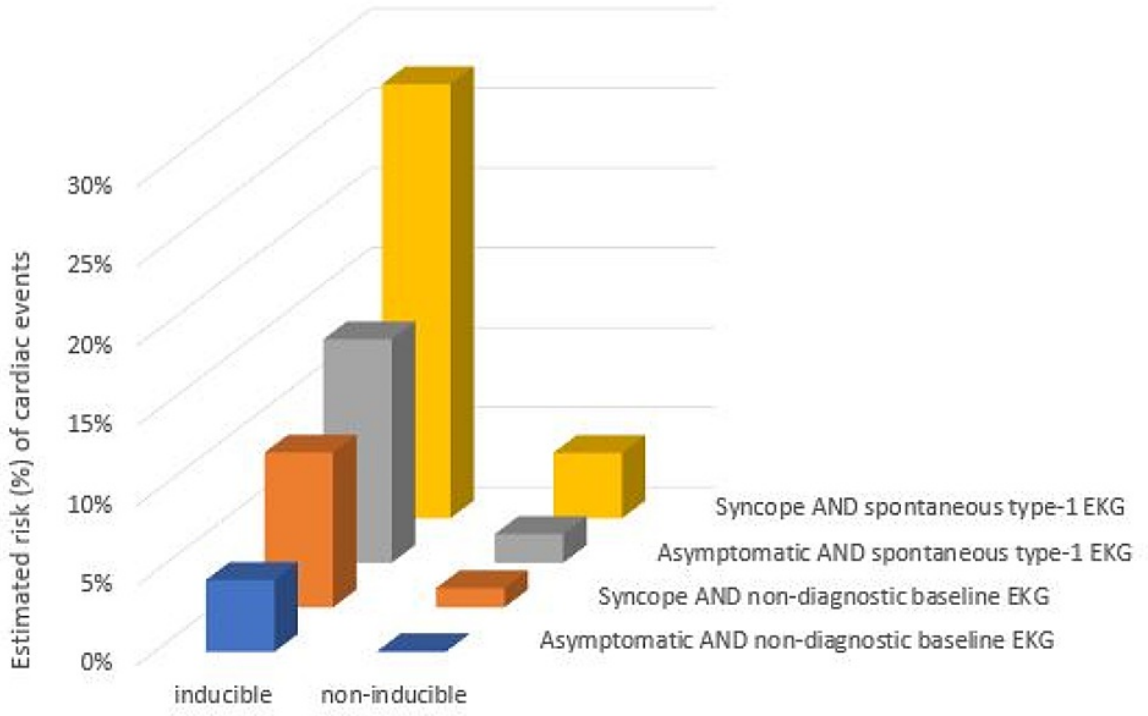

FIGURE 6: Probability of cardiac events during follow-up estimated by logistic regression analysis, according to the presence of symptoms, inducibility of arrhythmias, and type of baseline ECG.

Adapted from $[5,7]$.

There are three different types of Brugada EKG patterns: type 1, type 2, and type 3 (Figure 7). They can be distinguished by analyzing the ST segments in the V1-V2 precordial leads. In type 1, the ST segment is described as an elevated coved pattern with a high takeoff point ( $2 \mathrm{~mm}$ elevation from baseline) with no identifiable r', which is followed by a negative and symmetric T-wave. In type 2, the ST segment is noted to have a saddleback appearance, with a J-point $2 \mathrm{~mm}$ elevated from baseline and the terminal portion of the ST segment $1 \mathrm{~mm}$ above baseline. Type 3 is similar to type 2, except the ST segment is less than $1 \mathrm{~mm}$ above the baseline. In patients where Brugada syndrome is suspected without corresponding EKG, it may be possible to elicit these patterns by moving the right precordial leads superiorly to the second, third, or fourth intercostal space, as this has shown to increase the sensitivity of detecting the EKG pattern $[8,15,16]$. The basis for this observation is the current theory that tissue and microscopic changes located in the right ventricular outflow tract are responsible for the syndrome [7]. The presence of a type $1 \mathrm{EKG}$ pattern in conjunction with symptoms concerning for ventricular tachyarrhythmia or syncope is sufficient to make the diagnosis of Brugada syndrome. For type 2 EKG patterns, further workup is necessary to confirm the diagnosis.
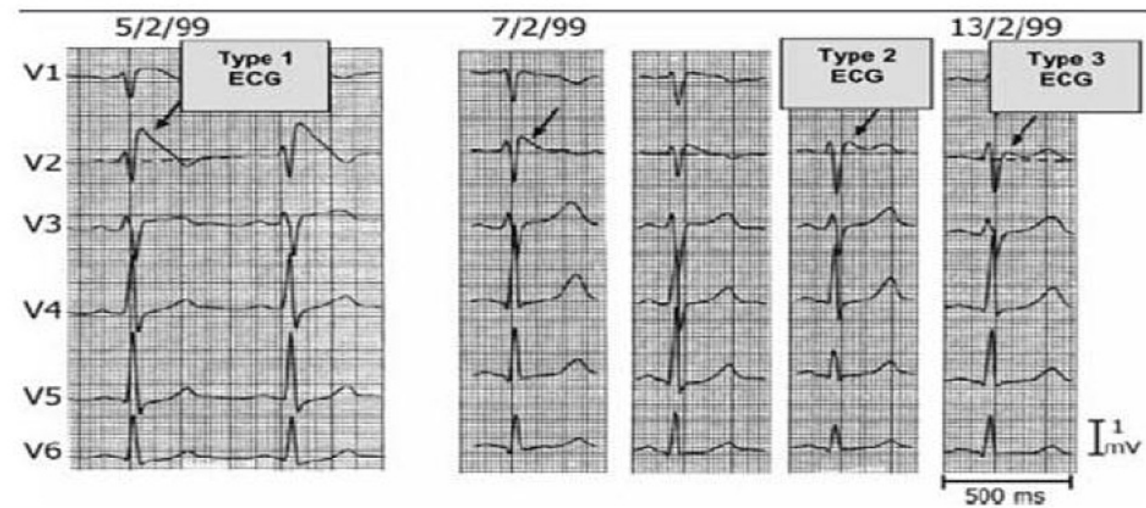

FIGURE 7: Brugada syndrome. Examples of type 1, type 2, and type 3 Brugada patterns.

Obtained and permission granted for reuse from [4]. 


\section{Cureus}

a drug challenge with a sodium channel blocker such as flecainide or procainamide (Figure 8) [8,16]. Many Brugada syndrome patients with family history components have genetic abnormalities in cardiac sodium channels that result in a decreased influx of sodium into the myocyte. Administration of a sodium channel blocker attempts to mimic this genetic abnormality. If the pharmacologic challenge elicits a type 1 EKG pattern in patients with cardiac arrest or unexplained syncope, current guidelines recommend implantable cardioverter-defibrillator (ICD) placement as first-line treatment (Class I recommendation) $[9,14]$. This would target the prevention of death from ventricular tachyarrhythmia, the major source of sudden death in Brugada syndrome patients. In patients where ICD placement is contraindicated or has failed, medical management with either quinidine or amiodarone can be added to the regimen [17,18]. If antiarrhythmic therapy fails, then catheter ablation can be considered [19]. For all patients presenting with type 1 EKG findings, genotyping including the SCN5A gene is recommended (Class IIb recommendation).

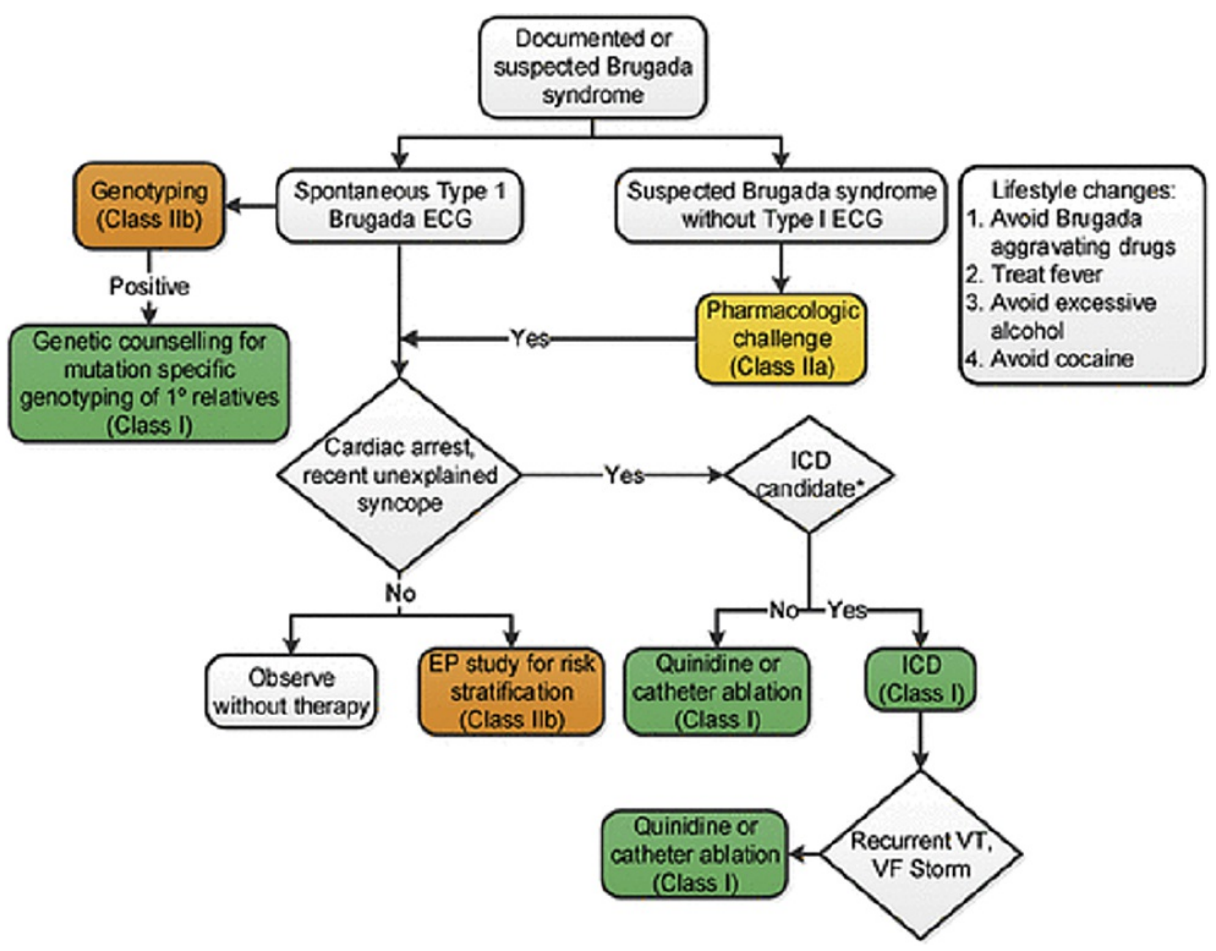

FIGURE 8: Suspected Brugada syndrome. Guideline-directed workup and primary prevention.

Obtained and permission granted for reuse from [9].

ICD, implantable cardioverter-defibrillator; EP, electrophysiology; VT, ventricular tachycardia; VF, ventricular fibrillation.

\section{Conclusions}

These cases illustrate the importance of maintaining an appropriate suspicion for Brugada syndrome in young patients with minimal ischemic risk factors. We discussed a guideline-directed algorithmic workup in suspicious individuals. Stratifying patients based on the presence of symptoms, history of tachyarrhythmias, and EKG findings before and after drug challenge allows physicians to guide further management of these patients.

\section{Additional Information \\ Disclosures}

Human subjects: Consent was obtained or waived by all participants in this study. Conflicts of interest: In compliance with the ICMJE uniform disclosure form, all authors declare the following: Payment/services info: All authors have declared that no financial support was received from any organization for the submitted work. Financial relationships: All authors have declared that they have no financial relationships at present or within the previous three years with any organizations that might have an interest in the submitted work. Other relationships: All authors have declared that there are no other relationships or activities that could appear to have influenced the submitted work. 


\section{References}

1. Imazio M, Gaita F, LeWinter M: Evaluation and treatment of pericarditis: a systematic review . JAMA. 2015, 314:1498-506. 10.1001/jama.2015.12763

2. Thygesen K, Alpert JS, Jaffe AS, Chaitman BR, Bax JJ, Morrow DA, White HD: Fourth universal definition of myocardial infarction (2018). J Am Coll Cardiol. 2018, 72:2231-64. 10.1016/j.jacc.2018.08.1038

3. Gehi AK, Duong TD, Metz LD, Gomes JA, Mehta D: Risk stratification of individuals with the Brugada electrocardiogram: a meta-analysis. J Cardiovasc Electrophysiol. 2006, 17:577-83. 10.1111/j.15408167.2006.00455.x

4. Antzelevitch C: Brugada syndrome. Pacing Clin Electrophysiol. 2006, 29:1130-59. 10.1111/j.15408159.2006.00507.x

5. Brugada J, Brugada R, Brugada P: Determinants of sudden cardiac death in individuals with the electrocardiographic pattern of Brugada syndrome and no previous cardiac arrest. Circulation. 2003, 108:3092-6. 10.1161/01.CIR.0000104568.13957.4F

6. Erickson CC, Salerno JC, Berger S, et al.: Sudden death in the young: information for the primary care provider. Pediatrics. 2021, 148:e2021052044. 10.1542/peds.2021-052044

7. Benito B, Brugada J, Brugada R, Brugada P: Brugada syndrome. Rev Esp Cardiol Engl Ed. 2009, 62:12971315. 10.1016/S1885-5857(09)73357-2

8. Antzelevitch C, Brugada P, Borggrefe M, et al.: Brugada syndrome: report of the second consensus conference: endorsed by the Heart Rhythm Society and the European Heart Rhythm Association. Circulation. 2005, 111:659-70. 10.1161/01.CIR.0000152479.54298.51

9. Al-Khatib SM, Stevenson WG, Ackerman MJ, et al.: 2017 AHA/ACC/HRS guideline for management of patients with ventricular arrhythmias and the prevention of sudden cardiac death: executive summary: a report of the American College of Cardiology/American Heart Association Task Force on Clinical Practice Guidelines and the Heart Rhythm Society. Heart Rhythm. 2018, 15:e190-252. 10.1016/j.hrthm.2017.10.035

10. Benito B, Sarkozy A, Mont L, et al.: Gender differences in clinical manifestations of Brugada syndrome . J Am Coll Cardiol. 2008, 52:1567-73. 10.1016/j.jacc.2008.07.052

11. Priori SG, Napolitano C, Gasparini M, et al.: Natural history of Brugada syndrome: insights for risk stratification and management. Circulation. 2002, 105:1342-7. 10.1161/hc1102.105288

12. Cardoso A, Faria B, Von Hafe P, et al.: Family screening in Brugada syndrome patients. Eur Heart J. 2021, 42:10.1093/eurheartj/ehab724.0635

13. Wallace ML, Ricco JA, Barrett B: Screening strategies for cardiovascular disease in asymptomatic adults . Prim Care. 2014, 41:371-97. 10.1016/j.pop.2014.02.010

14. Brugada J, Campuzano O, Arbelo E, Sarquella-Brugada G, Brugada R: Present status of Brugada syndrome: JACC state-of-the-art review. J Am Coll Cardiol. 2018, 72:1046-59. 10.1016/j.jacc.2018.06.037

15. Batchvarov VN, Govindan M, Macfarlane P, Camm AJ, Behr ER: Diagnostic utility of bipolar precordial leads during ajmaline testing for suspected Brugada syndrome. Heart Rhythm. 2010, 7:208-15. 10.1016/j.hrthm.2009.10.005

16. Sangwatanaroj S, Prechawat S, Sunsaneewitayakul B, Sitthisook S, Tosukhowong P, Tungsanga K: New electrocardiographic leads and the procainamide test for the detection of the Brugada sign in sudden unexplained death syndrome survivors and their relatives. Eur Heart J. 2001, 22:2290-6. 10.1053/euhj.2001.2691

17. Priori SG, Wilde AA, Horie M, et al.: HRS/EHRA/APHRS expert consensus statement on the diagnosis and management of patients with inherited primary arrhythmia syndromes: document endorsed by HRS, EHRA, and APHRS in May 2013 and by ACCF, AHA, PACES, and AEPC in June 2013. Heart Rhythm. 2013, 10:193263. 10.1016/j.hrthm.2013.05.014

18. Belhassen B, Glick A, Viskin S: Efficacy of quinidine in high-risk patients with Brugada syndrome . Circulation. 2004, 110:1731-7. 10.1161/01.CIR.0000143159.30585.90

19. Fernandes GC, Fernandes A, Cardoso R, Nasi G, Rivera M, Mitrani RD, Goldberger JJ: Ablation strategies for the management of symptomatic Brugada syndrome: a systematic review. Heart Rhythm. 2018, 15:1140-7. 10.1016/j.hrthm.2018.03.019 